\title{
Measuring data credibility and medical coding: a case study using a nationwide Portuguese inpatient database
}

\author{
Julio Souza $^{1,2}$ (D) Diana Pimenta $^{1}$ (D) Ismael Caballero $^{3}$ (D) $\cdot$ Alberto Freitas $^{1,2}$ (D
}

Published online: 12 June 2020

C) Springer Science+Business Media, LLC, part of Springer Nature 2020

\begin{abstract}
Some countries have adopted the diagnosis-related groups (DRG) system to pay hospitals according to the number and complexity of patients they treat. Translating diseases and procedures into medical codes based on international standards such as ICD-9-CM or ICD-10-CM/PCS is at the core of the DRG systems. However, certain types of coding errors undermine this system, namely, upcoding, in which data is manipulated by deliberately using medical codes that increase patient's complexity, resulting in higher reimbursements. In this sense, ensuring data credibility in the context of upcoding is critical for an effectively functioning DRG system. We developed a method to measure data credibility in the context of upcoding through a case study using data on pneumonia-related hospitalizations from six public hospitals in Portugal. Frequencies of codes representing pneumonia-related diagnosis and comorbidities were compared between hospitals and support vector machine models to predict DRGs were employed to verify whether codes with discrepant frequencies were related to upcoding. Data were considered not credible if codes with discrepant frequencies were responsible for increasing DRG complexity. Six pneumonia-related diagnoses and fifteen comorbidities presented a higher-than-expected frequency in at least one hospital and a link between increased DRG complexity, and these targeted codes was found. However, overall credibility was very high for nearly all conditions, except for renal disease, which presented the highest percentage of potential upcoding. The main contribution of this paper is a generic and reproducible method that can be employed to monitor data credibility in the context of upcoding in DRG databases.
\end{abstract}

Keywords Data quality · Diagnosis-related groups · Clinical coding · Hospital administration · Data credibility $\cdot$ Support vector machine

Electronic supplementary material The online version of this article (https://doi.org/10.1007/s11219-02009504-3) contains supplementary material, which is available to authorized users.

Julio Souza

juliobsouza@med.up.pt

Extended author information available on the last page of the article 


\section{Background}

In the Portuguese hospital management sector, all information concerning the patient's diseases, health status, disease progression, procedures, and treatments are routinely reported in health records, discharge notes, pathology, and surgical reports (Alonso et al. 2019). All this largely unstructured information is abstracted and translated into standard clinical codes representing the patient's diagnoses and procedures according to the International Classification of Diseases, Clinical Modification, ninth and tenth revisions (ICD-9-CM and ICD-10CM) (Centers for Medicare and Medicaid Services 2014; Centers for Medicare and Medicaid Services 2019). Structured-coded clinical data will be further grouped into diagnosis-related groups (DRGs), generating a database to be used in several subdomains, ranging from healthcare management and decision-making (including resource use monitoring and billing) to research (discovering disease tends) (Alonso et al. 2019). In Portugal, the DRG system currently in use is the all-patient refined diagnosis-related groups (hereafter APR-DRG) (Administração Central do Sistema de Saúde 2014), which groups hospital cases, here referred as episodes, into specific clusters of patients (APR-DRGs) with similar resource use and costs (Averill et al. 2013; Mathauer and Wittenbecher 2013). The construction of APR-DRG is performed through a complex flowchart-based method supported by a specific software application (“APR-DRG grouper") that considers clinical data from each inpatient episode as inputs and assigns an APR-DRG for that episode based mostly on the clinical codes representing medical diagnoses and inpatient procedures present in the data, among other variables such as age, sex, and discharge status (Averill et al. 2013).

Each APR-DRG represents the patient's reason for hospital admission, either in terms of disease or procedure performed, and is usually associated with a predefined, standard price (Mathauer and Wittenbecher 2013). In fact, the algorithm assigns a severity of illness (SOI) level to each APR-DRG at the end of the classification process, which is a score ranging from one to four (1 minor, 2 moderate, 3 major, 4 extreme), representing increasing reimbursement demands for patients into the same APR-DRG (Averill et al. 2013). Secondary diagnoses representing comorbidities and complications are what typically drive SOI assignment, being thus relevant clinical features in the APR-DRG system (Aiello and Roddy 2017). At this point, it is necessary to recall that the Portuguese government pays hospitals according to an established list of reimbursement rates that are linked to each APR-DRG and SOI level (Ministério da Saúde 2017). Therefore, failures in the data representing codes of diseases and procedures will undoubtedly have an economic impact on hospitals.

\subsection{Problem and case study}

In Portugal, the process of creating the databases to feed the APR-DRG system relies on clinical coding, in which trained medical coders are in charge to interpret medical records and translate all patient's clinical information into diagnosis and procedure codes from the ICD-9$\mathrm{M}$ and ICD-10-CM lists (Ministério da Saúde 2017). Figure 1 shows the information flow and data lifecycle related to the APR-DRG system, from patient's documentation to the creation of APR-DRG data.

Therefore, the credibility of all codes entered for each episode is critical to ensure a good performance of the APR-DRG system. The subjective generation of codes when interpreting medical records, however, can lead to data quality problems, namely, loss of objectivity and data credibility (Strong et al. 1997). This is of great concern in the context of DRG systems, as 
several consequences could arise from the wide range of types of coding errors, including negative impacts on hospital finances and decreased reliability for reusing this data for clinical research and healthcare management. The most common types of coding errors include selecting the wrong principal diagnosis, omitting additional codes, and choosing less specific diagnosis and procedure codes (Reid et al. 2000). There are also deliberate coding errors, with the most common being denominated in the literature as upcoding, in which coders purposely miscode patient data to group episodes into higher-complexity DRGs to receive higher reimbursements (Dafny 2005; Silverman and Skinner 2004). For instance, Psaty et al. (1999) found that just adding a heart failure code as a complication to conditions such as acute myocardial infarction, pneumonia, or gastrointestinal hemorrhage would increase reimbursement to hospitals by over $\$ 3000.00$ on average. Exploring medical records to extract the most lucrative codes, including changes between the principal and secondary diagnosis, looking for reimbursable conditions, and exaggerating the choice of codes without supportive evidence in the patient's record are examples of upcoding practices (Pongpirul and Robinson 2013).

The main goal of this article was to assess the credibility of coded clinical data used in the APR-DRG system. We considered the definitions of data credibility according to ISO/IEC 25012 , which states that credibility refers to "the degree to which data has attributes that are regarded as true and believable by users in a specific context of use. Credibility includes the concept of authenticity (the truthfulness of origins, attributions, commitments)" (ISO/IEC 25012 2006). In our case study, due to feasibility reasons and as a pilot for the methodology, upcoding was the only type of coding error explored. Therefore, in this study, we will assess data credibility in the specific context of upcoding. Moreover, for the sake of usability and understandability, our case study was limited to pneumonia-related conditions, which is a diagnosis area that has been regarded as a target of upcoding in the literature (Sjoding et al.

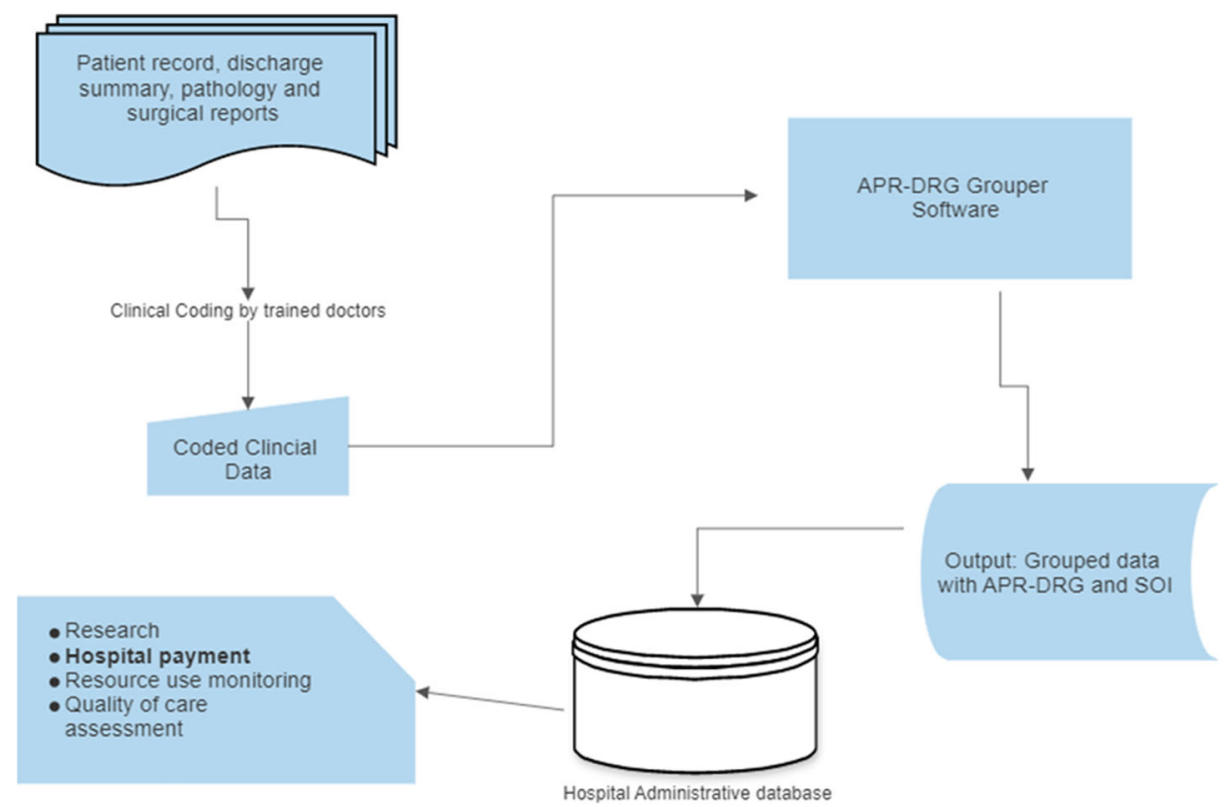

Fig. 1 The information flow and data lifecycle related to the APR-DRG system, from patient's documentation to the creation of APR-DRG data 
2015; Hebert et al. 2005). The case study also includes comorbidities, which are secondary diagnoses representing the patient's preexisting conditions underlying the primary diagnosis. Comorbidities in the context of upcoding are not only relevant because they typically drive the determination of patient's severity within the APR-DRG algorithm, regardless of the medical field (Aiello and Roddy 2017), but also because adding more diagnoses such as comorbidities to make the patient's condition look more complicated is a typical upcoding practice (Pongpirul and Robinson 2013). It is important to highlight that the only way to confirm upcoding is by auditing the health records that originated all coded data. However, the audit is a very high resource and time-consuming process and could not comprise the totality of data. Rather, it is internally performed in random clinical processes (Barros and Braun 2017).

This article is the extended version of the paper presented at the "12th International Conference on the Quality of Information and Communications Technology," which described a method to measure the credibility of data used for APR-DRG classification in the context of upcoding (Pimenta et al. 2019). In the absence of historical audit data and medical records, the main contribution of our solution is to provide a fairly automatized, generic, and reproducible method to measure data credibility for APR-DRG classification in the context of upcoding. In particular, we restricted our case study to upcoding and pneumonia-related hospitalizations, but since our method is based on well-established statistical and machine learning methods, we reinforce that this method can be employed to measure data credibility in the context of other types of coding errors and for other clinical domains.

\section{State of the art}

Credibility can be defined as "the overall plausibility or reliability of the data and the degree to which data are perceived as credible can be influenced by validation from primary sources and the alignment of the data with current medical knowledge and user-perceived reality" (Feder 2018). Validation rules can be used to determine whether data values appear to be credible in terms of clinical or other natural perspectives. Validity checks to determine if specific elements within patient data were likely to be true include looking for elements with values that were outside biologically plausible ranges, changed implausibly over time (Weiskopf and Weng 2013). Study design involving multiple sites and databases can also be useful to evaluate data credibility (Weiskopf and Weng 2013). Lau et al. (2011) assessed data credibility by comparing inputted data from electronic health records with records and claim data held in other official databases. Frequency distribution of data can also be compared with other sources, such as nationally published disease incidence or prevalence rates, to assess credibility (Rea et al. 2013).

In this study, the presence of upcoding is the aspect undermining data credibility. Methods for upcoding detection in hospital data have been mixed. Most studies have employed chart review, which consists in recording and regrouping the original medical records that originated coded data to verify whether the newly coded episodes would be grouped into a different DRG (Hsia 1990; Hsia et al. 1992; Goodpasture et al. 2004; Reid et al. 2005) and whether these changes occurred by means of increasing or decreasing DRG complexity.

In the absence of the original medical records, authors differed in the methodology for upcoding detection. Silverman and Skinner (2004) estimated the levels of upcoding by calculating the ratio of the number of discharges grouped into a DRG representing respiratory infections and inflammations with complications by the sum of cases grouped into all other 
DRGs representing respiratory ailments. This ratio was assessed over time and compared by the type of hospital ownership (not-for-profit, for-profit, and government hospitals). Hebert et al. (2005) assumed that a DRG commonly associated with a secondary diagnosis of pneumonia and influenza would pay less than if it had been reported as a principal diagnosis. In this sense, the authors investigated the DRG distribution in all claims with this condition listed as principal or secondary diagnosis over time.

Aelvoet et al. (2009) employed fixed effects models to assess deviant coding behavior at the hospital level by examining the linear evolution over time of certain outcomes (e.g., number of days the patient spent in the hospital) of a set of diseases and conditions that are susceptible to increase the complexity of an episode. For cases of deviant coding, these targeted conditions were marked to direct the audit toward fraud-suspected cases. A Norwegian study also employed fixed effects models to assess variations in the number of patients treated within the different DRGs over time, taking into account changes in the national average cost per DRG offered to Norwegian hospitals during the studied period (Januleviciute et al. 2016). Fixed effects models were also employed by Dafny (2005) and Barros and Braun (2017) to investigate the existence of potential upcoding cases in hospital databases. In a similar assumption, an Italian study used a difference-in-differences method to test whether hospitals responded to changes in DRG prices, including by means of upcoding, and defined as the proportion of patients with complications (Di Giacomo et al. 2017).

The use of machine learning for upcoding detection in hospital databases was employed by Luo and Gallagher (2010) and Souza et al. (2018), also in the absence of original medical records or audit data, which is the case of our study. In both studies, an unsupervised method was employed by comparing different distributions of DRGs in several hospitals in order to filter data to be monitored for potential upcoding.

\section{Methods}

\subsection{Generic method}

To the best of our knowledge, this is the first study to measure the credibility of clinical data in the context of upcoding. More specifically, data credibility can be understood as the level of certitude that episodes in the datasets are free of upcoding, thereby representing the actual patient's clinical condition and severity and thus grouped into an APR-DRG accordingly. Considering the absence of the original medical records and past audit data, an unsupervised method based on statistical and machine learning techniques was designed and summarized in Fig. 2. Further details concerning the method for measuring data credibility are explained in Section 3.3 .

\subsection{Description of the data source}

Data used in this study were extracted from the Portuguese National Hospital Morbidity Database, which contains inpatient and outpatient discharge data from all public hospitals from the National Health System (NHS) in Portugal. The Portuguese National Hospital Morbidity Database contains a comprehensive list of administrative, clinical, and demographic data fields. The main data attributes that are used for APR-DRG grouping were collected and considered in the method for data credibility measurement: principal and secondary diagnoses, 
- Data quality measure name

- Measurement function

- Quality measure elements

- Measurement unit

- Measurement method
Credibility of hospital clinical data for APR-DRG grouping

$[1-(A / B)]^{*} 100$

$A=$ Number of pneumonia-related episodes in the dataset that are considered potential cases of upcoding

$B=$ Total number of pneumonia-related episodes in the dataset

Percent

Inter-hospital variation in coding using Chi-squared test corrected for multiple comparisons.

SVM models for APR-DRG classification to assess possibility of upcoding.

- Analysis model
Codes with a significantly higher frequency in at least one hospital were targeted as potential upcoding candidades. We considered p-values lower than Bonferroni threshold for multiple testing correction to identify these codes with abnormal frequencies.

Using SVM models, we further evaluated the influence of candidate codes by assessing APR-DRG classification with and without the said codes.

Fig. 2 Summary of unsupervised method based on statistical and machine learning techniques

as well as inpatient procedures, specified according to the ICD-9-CM; discharge status, which is represented by a standard code indicating the patient's fate after the discharge, including departure to home, death and transfers to another hospital, or specialized services; and sex and age.

In this study, we analyzed data for 2015, which was the last year when ICD-9-CM was still used for coding diseases in Portugal. From 2016 onward, episodes were either coded in ICD-9$\mathrm{CM}$ or in the newest revision, ICD-10-CM. Thus, we did not include more recent years to avoid further bias related to the transition between ICD versions. Moreover, in order to minimize the bias introduced by differences in the complexity of hospitals, we restricted our evaluation to six large hospitals with similar capacity and complexity, based on a standard categorization defined by the Portuguese NHS (Administração Central do Sistema de Saúde 2019). Furthermore, we selected all episodes with a principal or secondary diagnosis code from the ICD-9-CM list comprised in the interval of 480-488, which corresponds to pneumonia and influenza diagnoses. Comorbidities were selected according to the enhanced ICD9-CM Charlson's definitions (Quan et al. 2005).

Since inpatient data used in this study were anonymized and only contained the discharge year, diagnosis and procedure codes, sex, age, discharge status, and an arbitrary episode identification number, there was no need for ethical approval and no data privacy law was violated.

\subsection{Particularization of the method for data credibility measurement}

In our method, the starting point to detect potential upcoding is by tracking diagnosis codes with an abnormally high frequency in a given hospital relatively to other hospitals. Data are considered not credible when an episode presents a diagnosis code with an abnormally high frequency in the hospital it was admitted to and if that code alone is responsible for increasing APR-DRG complexity and, thereby, hospital reimbursement. 
The identification of codes with discrepant frequencies occurred in three phases. First, we compared hospitals regarding APR-DRG distributions using Chi-square test with Bonferroni correction for multiple comparisons, so that the proportion of episodes grouped into a specific APR-DRG in a given hospital is compared against all other facilities. Second, we compared APR-DRG distributions between hospitals, but now considering the SOI level and restricting to the APR-DRGs targeted in the first phase, which correspond to those APR-DRGs with an abnormally high frequency in at least one hospital. In the last phase, the same statistical test was employed to compare hospitals with regard to the frequency of individual codes representing comorbidities and pneumonia-related diagnoses among episodes grouped into the APR-DRGs targeted in the second phase. Following this analysis, all comorbidities and pneumonia codes that present a significantly higher-than-expected frequency in at least one hospital were targeted, as they might have led to potential cases of upcoding.

To investigate whether the targeted codes could be associated with upcoding, we employed support vector machine (SVM) to build a model for APR-DRG classification. The SVM algorithm was introduced by Vapnik (1995) and is a consistently good classifier, being widely used as a classification method (Chu et al. 2008). Another important advantage of using SVM is that it can overcome high-dimensionality issues (Chu et al. 2008; Verplancke et al. 2008), which is the case of the APR-DRG classification problem. Other advantages of SVM include high accuracy, avoids overfitting, and accuracy and performance are independent of the number of features, and it presents a good generalization ability (Singh et al. 2016). Two SVM classification models were built to simulate the APR-DRG system:

- SVM-ONE to predict one out of the 17 APR-DRGs defined for classifying diseases or disorders of the respiratory system, based upon the APR-DRG version 31 (Averill et al. 2013).

- SVM-TWO to determine the SOI level. The APR-DRG assigned by SVM-ONE is used as an input variable for SVM-TWO.

\subsection{Implementation details for SVM-ONE and SVM-TWO}

Regarding the specification of variables for SVM-ONE, we included age as continuous attribute, sex, and discharge status as categorical attributes and all the existing ICD-9-CM diagnosis and procedure codes in the dataset. As SOI classification does not take into account sex and discharge status, SVM-TWO only included age as a continuous attribute and the APRDRG itself as categorical attribute and all diagnosis and procedure codes. Each ICD-9-CM code was treated as an individual binary variable (" 1 " if the episode reported the code, " 0 " otherwise).

Using the constructed SVM models, we performed a sensitivity analysis to discover the role of each discrepant code on APR-DRG classification by removing the code from the original episode and assessing APR-DRG classification changes. If the exclusion of the code alone moves the episode to a lower complexity APR-DRG, then the episode is labeled as a potential case of upcoding. It is important to highlight that this verification can only be performed for secondary diagnosis codes, since the principal diagnosis is mandatory for APR-DRG grouping, so the exclusion of the principal diagnosis in the sensitivity analysis would simply invalidate APR-DRG grouping. Thus, to assess the role of principal diagnoses on APR- 
DRG grouping, we simply studied the frequency distributions of the codes within each APRDRG, instead of directly applying the SVM approach. Finally, as defined in Fig. 1, we estimated the percentage of potential cases of upcoding from the total number of episodes, for each hospital and disease (pneumonia-related condition or comorbidity), to calculate the levels of credibility.

Training and testing phases of SVMs, as well as sensitivity analyses, were performed using a Java program, which run in a Java Runtime Environment (JRE) 1.8.0_221 in combination with Java Standard Edition Development Kit (JDK) 1.8.0_221 and an open-source Weka library for Java, version 3.8.0. The integrated development environment used for implementing the Java code was Eclipse Mars 2. The running environment was a Windows $10 \mathrm{PC}$ with a CPU Intel $1.80 \mathrm{GHz}$ and $16 \mathrm{~GB}$ of RAM. We considered the algorithm for training the SVM models introduced by Platt (1998) and denominated sequential minimal optimization available in the Weka library for Java, considering default parameters, as detailed in the library's documentation.

\subsection{Sensibility of the results: quality of the SVM models used for data credibility measurement}

Data of 487,156 hospitalizations were used for training and testing the SVM models, which corresponds to all inpatient episodes due to respiratory system disorders occurred in the period of 2011-2015 in public Portuguese hospitals which were statically valid. This latter criterion refers to episodes within the length of stay interval determined for the APR-DRG they were assigned to (Ministério da Saúde 2017). Episodes due to respiratory system diseases were identified based upon a principal diagnosis list defined for the APR-DRG system (Averill et al. 2013). The chosen period comprises all available APRDRG data coded according to the ICD-9-CM. Therefore, we considered this specific period to have access to the most comprehensive dataset to construct SVM models for APR-DRG prediction.

We trained the SVM models on two-thirds of the dataset and tested their performance on the remaining third. As evaluation metrics of the goodness of the models, we considered the percentage of correctly classified cases and weighted precision and recall. Estimates of precision and recall for each class (APR-DRG for SVM-ONE and SOI level for SVM-TWO) were weighed by the number of instances within each class, providing the weighted precision and recall, as follows:

$$
\text { Weighted precision } / \text { recall }=\frac{\sum_{i}^{n} P_{C i}{ }^{*} C_{i}}{\sum_{i}^{n} C i}
$$

where $P_{C i}$ is the precision or recall for class $i(i=1, \ldots, 17$ for SVM-ONE and $i=1, \ldots, 4$ for SVM-TWO) and $C_{i}$ is the number of instances in class $i$. Weighting by class size provides a better estimate of overall performance of the SVM models, since the APRDRG or SOI (classes) frequencies can be very different (e.g., certain APR-DRGs are much more frequent in the real world than others, and less severe episodes tend to be more prevalent).

We further tested the SVM models on unseen ICD-9-CM data from the year 2016 and estimated the percentage of correctly classified cases to add critical validation to the models in terms of generalization capacity. 


\section{Results}

\subsection{Performance of SVM models (method for data credibility measurement)}

Regarding the performance of the SVM models, considering the SVM-ONE, weighted recall and precision were both 0.994 , and the percentage of correctly classified cases was $99.4 \%$. For the second model, SVM-TWO, overall weighted recall and precision were both 0.893 , with a percentage of correctly classified cases of $89.3 \%$. When tested in ICD-9-CM data from 2016 (92,475 episodes), we used SVM-ONE and SVM-TWO simultaneously and compared with the original APR-DRG and SOI classifications. We verified that the SVM models together presented a high capacity of generalization, with a percentage of correctly classified cases of $88.4 \%$. Supplementary Table 1 presents the detailed evaluation metrics results after the testing phase, for each possible class (APR-DRG and SOI levels).

\subsection{Data credibility concerning upcoding}

To detect diagnosis codes that could have been target of upcoding in the evaluated hospitals, we applied statistical tests in three phases. In the first phase, frequencies of APR-DRGs were compared between hospitals using Chi-square test with Bonferroni correction for multiple comparisons. We only compared frequencies of APR-DRGs with enough episodes to perform a robust Chi-square test, as defined in Yates et al. (1999) (contingency table with more than $80 \%$ of the cells presenting expected counts equal to five or more; the remaining $20 \%$ can present an expected count below five, as long as none are less than one). Significantly higherthan-expected frequencies were found for five APR-DRGs: APR-DRGs 120 (major respiratory and chest procedures), 130 (respiratory system diagnosis with ventilator support $96+\mathrm{h}$ ), 137 (major respiratory infections and inflammations), 138 (bronchiolitis and RSV pneumonia), and 139 (other pneumonia). Detailed results of the first phase analysis are presented in supplementary Table 2. Our hypothesis is that discrepant frequencies of APR-DRGs between hospitals might be the first indicator of upcoding. However, as patient complexity and prices are mostly determined by APR-DRGs in combination with SOI, we further applied the same method in a second phase to compare the frequencies of the five discrepant APR-DRGs but now considering the SOI level. Only APR-DRGs and SOI levels with enough episodes to perform Chi-square test were further considered, reducing the analyses to three APR-DRGs, 137, 138 and 139, and combined with their respective SOI levels. All three APR-DRGs presented a significantly higher-than-expected frequency of a given SOI level in at least one hospital. Results of the second phase analysis are presented in supplementary Table 3.

Finally, in order to discover which codes might have been upcoded, we also compared hospitals with regard to the frequencies of individual diagnosis codes in a third phase analysis. To assess this inter-hospital variation in coding, for each discrepant APR-DRG, the frequency of each pneumonia-related diagnosis and comorbidity code was compared across hospitals using the same Chi-square-based approach. A total of six pneumonia-related diagnosis codes presented a significantly higher-than-expected frequency in at least one hospital: 482.42, methicillin-resistant pneumonia due to Staphylococcus aureus; 480.9, viral pneumonia, unspecified; 481, pneumococcal pneumonia; 482.9, bacterial pneumonia, unspecified; 485, bronchopneumonia, organism unspecified; and 486, pneumonia, organism unspecified. Regarding comorbidities, conditions with a significantly higher-than-expected frequency in at least one hospital were any malignancy, chronic pulmonary disease, renal disease, congestive 


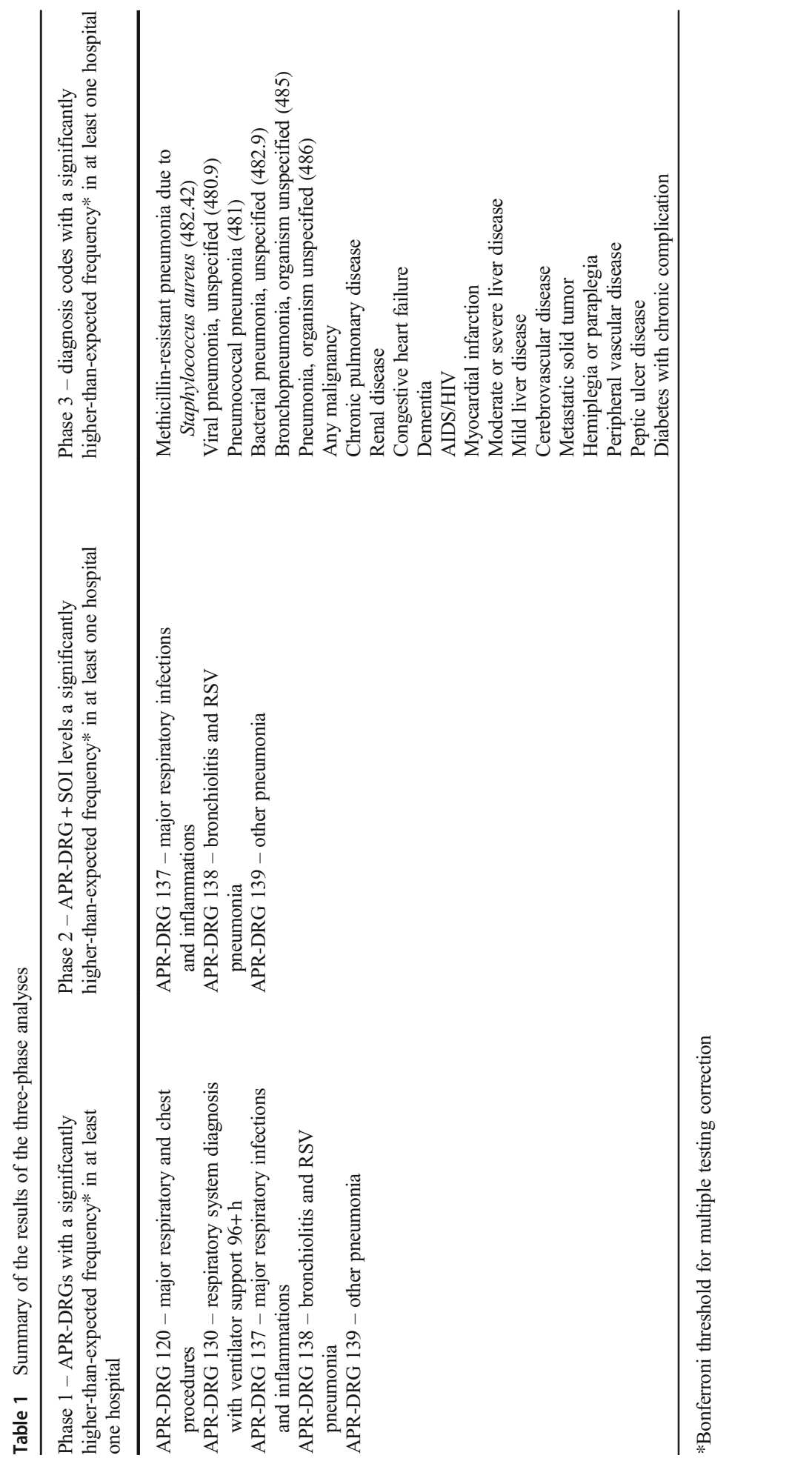


Table 2 Individual effects of each discrepant pneumonia-related diagnosis on APR-DRG classification

\begin{tabular}{|c|c|c|c|}
\hline & $\begin{array}{l}\text { Increased APR-DRG } \\
\text { complexity ( } n \text { of episodes) }\end{array}$ & $\begin{array}{l}\text { Decreased APR-DRG } \\
\text { complexity ( } n \text { of episodes) }\end{array}$ & $\begin{array}{l}\text { Total ( } n \text { of } \\
\text { episodes) }\end{array}$ \\
\hline \multicolumn{4}{|l|}{ Principal diagnosis } \\
\hline $\begin{array}{l}\text { Methicillin-resistant pneumonia due } \\
\text { to Staphylococcus aureus }\end{array}$ & 0 & 0 & 102 \\
\hline Viral pneumonia, unspecified & 0 & 53 & 54 \\
\hline Pneumococcal pneumonia & 1 & 483 & 509 \\
\hline Bacterial pneumonia, unspecified & 1 & 466 & 488 \\
\hline $\begin{array}{l}\text { Bronchopneumonia, organism } \\
\text { unspecified }\end{array}$ & 0 & 449 & 455 \\
\hline Pneumonia, organism unspecified & 1 & 6926 & 7059 \\
\hline Total & 3 & 8377 & 8667 \\
\hline \multicolumn{4}{|l|}{ Secondary diagnosis } \\
\hline $\begin{array}{l}\text { Methicillin-resistant pneumonia due } \\
\text { to Staphylococcus aureus }\end{array}$ & 3 & 0 & 64 \\
\hline Viral pneumonia, unspecified & 0 & 0 & 5 \\
\hline Pneumococcal pneumonia & 5 & 0 & 34 \\
\hline Bacterial pneumonia, unspecified & 26 & 0 & 78 \\
\hline $\begin{array}{l}\text { Bronchopneumonia, organism } \\
\text { unspecified }\end{array}$ & 3 & 0 & 39 \\
\hline Pneumonia, organism unspecified & 185 & 0 & 540 \\
\hline Total & 222 & 0 & 760 \\
\hline
\end{tabular}

heart failure, dementia, AIDS/HIV, myocardial infarction, moderate or severe liver disease, mild liver disease, cerebrovascular disease, metastatic solid tumor, hemiplegia or paraplegia, peripheral vascular disease, peptic ulcer disease, and diabetes with chronic complication.

Table 1 summarizes the results of the three-phase analyses, which resulted in a list of diagnosis codes with discrepant frequencies which could have been target of upcoding practices.

Table 3 Individual effects of each discrepant comorbidity on APR-DRG classification

\begin{tabular}{llll}
\hline Comorbidity & $\begin{array}{l}\text { Increased APR-DRG } \\
\text { complexity }(n \text { of } \\
\text { episodes) }\end{array}$ & $\begin{array}{l}\text { Decreased APR-DRH } \\
\text { complexity ( } n \text { of } \\
\text { episodes) }\end{array}$ & $\begin{array}{l}\text { Total ( } n \text { of } \\
\text { episodes })\end{array}$ \\
\hline
\end{tabular}

Myocardial infarction

Congestive heart failure

Peripheral vascular disease

Cerebrovascular disease

Dementia

Chronic pulmonary disease

Rheumatic disease

Mild liver disease

Diabetes without chronic complication $\quad 350$

Diabetes with chronic complication $\quad 44$

Hemiplegia

Renal disease

Any malignancy

Moderate or severe liver disease

Metastatic solid tumor

AIDS/HIV

Total

$\begin{array}{rr}89 & 2 \\ 830 & 1 \\ 23 & 7 \\ 247 & 5 \\ 130 & 0 \\ 143 & 1 \\ 29 & 2 \\ 30 & 7 \\ 350 & 1 \\ 44 & 2 \\ 67 & 2 \\ 3763 & 1 \\ 460 & 37 \\ 20 & 3 \\ 421 & 0 \\ 8 & 2 \\ 6654 & 0\end{array}$


In order to discover the role of the discrepant codes within the APR-DRG grouping process and thus investigate potential upcoding practices, we performed a sensitivity analysis using the SVM models to assess APR-DRG classification changes with and without the discrepant code. Table 2 summarizes the SVM results by indicating how many episodes were driven to a given APR-DRG by each one of the targeted pneumoniarelated codes, either when those were coded as principal or secondary diagnosis, whereas Table 3 presents the results for comorbidities, which are conditions that can only be coded as secondary diagnoses.

Diagnosis code "pneumonia, organism unspecified" occurred in 7059 episodes, and, from this total, it was responsible for allocating 6926 episodes to an APR-DRG of lower complexity (APR-DRG 139) when coded as principal diagnosis, whereas it placed 185 episodes (out of 540) in an APR-DRG of higher complexity (APR-DRG 137) when coded as secondary diagnosis (Table 2). In fact, this tendency was observed for five pneumonia-related conditions, as 222 episodes (out of 760) were allocated to a higher complexity APR-DRG by simply being coded as secondary diagnosis (Table 2). Moreover, we observed that comorbidities generally increased APR-DRG complexity. In fact, comorbidities alone drove 6654 into higher-paying APR-DRGs (Table 3).

Table 4 shows, for each hospital and disease (pneumonia-related diagnosis or comorbidity), the number of hospitalizations flagged as potential cases of upcoding after the application of our criteria and the respective credibility levels, which in turn consist in the proportion of episodes that are not labeled potential cases of upcoding. Episodes are labeled as a potential case of upcoding case if they present a discrepant code that alone increases APR-DRG complexity. A total of 2916 episodes across the evaluated hospitals were targeted as potential upcoding cases, with the impact size varying depending on the hospital and diagnosis code (see Table 4).

At the hospital level, the occurrence of potential cases of upcoding was proportionally small, reaching around $1 \%$ of the cases for most of the targeted codes. Consequently, according to our method, overall data credibility levels across hospital databases were very high, with values higher than $99 \%$ for the clear majority of the diseases. However, for certain specific codes, we found that credibility levels were substantially lower, namely, for renal disease, which was the condition with the highest percentage of upcoding in all hospitals. In fact, credibility levels concerning renal disease were particularly low for hospital A (83.5\%) and hospital F $(57.8 \%)$.

\section{Discussion of the findings}

The credibility of coded clinical data in hospital administrative databases is a critical issue in APR-DRG systems and in other contexts where this data can be productively reused, namely, health care funding, research, decision-making, and quality of care assessment. Despite several existing problems, healthcare administrative data have a lot of potentials to produce quality indicators and health research (Jarman et al. 1999; Scott et al. 2004), and understanding the process of medical coding through data mining techniques, which was the basis for our method, have already been explored in the literature (Spangler et al. 2002) and may be useful to provide insights on the quality of coded data. The focus of this paper was to measure data 
Table 4 Credibility levels in pneumonia-related hospitalization data, by diagnosis code

Hospital A

\begin{tabular}{llll}
\hline Disease & $\begin{array}{l}\text { Number of potential } \\
\text { cases of upcoding } \\
(n \text { of episodes })\end{array}$ & $\begin{array}{l}\text { Upcoding } \\
\text { proportion }(\%)\end{array}$ & $\begin{array}{l}\text { Credibility } \\
\text { level }(\%)\end{array}$ \\
\hline
\end{tabular}

Pneumococcal pneumonia 2

Bacterial pneumonia, unspecified

Pneumonia, organism unspecified

Myocardial infarction

Congestive heart failure

Cerebrovascular disease

Dementia

Chronic pulmonary disease

Mild liver disease

Diabetes without chronic complications

Diabetes with chronic complications

Hemiplegia or paraplegia

Renal disease

Any malignancy

Moderate or severe liver disease

Metastatic solid tumor

Overall (Mean)

Hospital B

Disease

Bacterial pneumonia, unspecified

Pneumonia, organism unspecified

Myocardial infarction

Congestive heart failure

Peripheral vascular disease

Cerebrovascular disease

Dementia

Chronic pulmonary disease

Rheumatic disease

Mild liver disease

Diabetes without chronic complications

Diabetes with chronic complications

Hemiplegia or paraplegia

Renal disease

Any malignancy

Moderate or severe liver disease

Metastatic solid tumor

Overall (Mean)

Hospital C

Disease

Pneumonia due to Haemophilus influenzae

Pneumococcal pneumonia

Pneumonia, organism unspecified

Myocardial infarction

Congestive heart failure

Peripheral vascular disease

Cerebrovascular disease

Dementia

Chronic pulmonary disease

1

6

5

77

22

12

5

2

19

5

1

121

33

2

7

\section{N}

Number of potential cases of upcoding ( $n$ of episodes) 1 4 7 59

3

21

17

5

1

2

26

4

4

478

41

1

10
Number of potential cases of upcoding ( $n$ of episodes) 1 1 6

$\begin{array}{ll}0.1 & 99.9 \\ 0 & 100 \\ 0.2 & 99.8 \\ 0.2 & 99.8 \\ 3.1 & 96.9 \\ 0.9 & 99.1 \\ 0.5 & 99.5 \\ 0.2 & 99.8 \\ 0.1 & 99.9 \\ 0.8 & 99.2 \\ 0.2 & 99.8 \\ 0 & 100 \\ 4.9 & 95.1 \\ 1.3 & 98.7 \\ 0.1 & 99.9 \\ 0.3 & 99.7 \\ 0.8 & 99.2\end{array}$
Upcoding proportion (\%)

\section{Credibility} level (\%)

100

99.9

99.8

98

99.9

99.3

99.4

99.8

100

99.9

99.1

99.9

99.9

83.5

98.6

100

99.7

$\begin{array}{ll}0.3 & 99.7 \\ 1.4 & 99.6\end{array}$

Upcoding Credibility proportion $(\%) \quad$ level $(\%)$

$0.1 \quad 99.9$

$\begin{array}{ll}0.1 & 99.9\end{array}$

$\begin{array}{ll}0.3 & 99.7\end{array}$

0.3
1.3

1.8
0.1

$0.1 \quad 99.9$

$0.7 \quad 99.3$

$\begin{array}{ll}0.3 & 99.7\end{array}$

$0.6 \quad 99.4$ 
Table 4 (continued)

Hospital A

Disease

Number of potential cases of upcoding ( $n$ of episodes)
Upcoding

proportion (\%)

Credibility

level $(\%)$

\begin{tabular}{llll}
\hline Rheumatic disease & 1 & 0.1 & 99.9 \\
Mild liver disease & 3 & 0.2 & 99.8 \\
Diabetes without chronic complications & 16 & 0.9 & 99.1 \\
Diabetes with chronic complications & 4 & 0.2 & 99.8 \\
Hemiplegia or paraplegia & 1 & 0.1 & 9.9 \\
Renal disease & 86 & 1 & 99.9 \\
Any malignancy & 18 & 0.1 & 95.1 \\
Moderate or severe liver disease & 2 & 0.3 & 99.9 \\
Metastatic solid tumor & 6 & 0.7 & 99.7 \\
Overall (Mean) & & 99.3
\end{tabular}

Hospital E

Disease

Number of potential cases of upcoding ( $n$ of episodes)

Pneumonia, organism unspecified

Myocardial infarction

Congestive heart failure

Peripheral vascular disease

Cerebrovascular disease

Dementia

Chronic pulmonary disease

Rheumatic disease

Diabetes without chronic complications

Diabetes with chronic complications

Hemiplegia or paraplegia

Renal disease

Any malignancy

Moderate or severe liver disease

Metastatic solid tumor

Overall (Mean)

Hospital F

Disease

Methicillin resistant pneumonia due to Staphylococcus aureus

Bacterial pneumonia, unspecified

Bronchopneumonia, organism unspecified

Pneumonia, organism unspecified

Myocardial infarction

Congestive heart failure

Peripheral vascular disease

Cerebrovascular disease

Dementia

Chronic pulmonary disease

Rheumatic disease

Mild liver disease

Diabetes without chronic complications

Diabetes with chronic complications

Hemiplegia or paraplegia

Renal disease

Any malignancy

\section{Upcoding \\ proportion (\%)}

Credibility level $(\%)$

3

6

24

2

12

5

8

3

16

2

8

85

23

1

7

Number of potential cases of upcoding ( $n$ of episodes)

1

0.2

0.3

1.2

0.1

0.6

0.3

0.4

0.2

0.8

0.1

0.4

4.4

1.2

0.1

0.4

0.7

\section{Upcoding proportion (\%)}

0

0.1

0

0.1

0.5

4.6

0.1

1.8

1.1

0.8

0.1

0.2

2.9

0.2

0.5

42.2

1085

49
99.8

99.7

98.8

99.9

99.4

99.7

99.6

99.8

99.2

99.9

99.6

95.6

98.8

99.9

99.6

99.3

Credibility level (\%) 
Table 4 (continued)

Hospital A

\begin{tabular}{llll}
\hline Disease & $\begin{array}{l}\text { Number of potential } \\
\text { cases of upcoding } \\
(n \text { of episodes })\end{array}$ & $\begin{array}{l}\text { Upcoding } \\
\text { proportion }(\%)\end{array}$ & $\begin{array}{l}\text { Credibility } \\
\text { level }(\%)\end{array}$ \\
\hline $\begin{array}{l}\text { Metastatic solid tumor } \\
\text { Overall (Mean) }\end{array}$ & 10 & 0.4 & 99.6 \\
\hline
\end{tabular}

credibility in the context of upcoding, using a case study on pneumonia-related hospitalizations. In the absence of medical records to verify the sources that originated coded data, as well as lack of historical audit data, we used an approach based on statistical analysis and machine learning to blindly detect potential upcoding. Our method only requires data on APR-DRG and the respective variables used for APR-DRG classification, namely, codes of diagnoses and procedures.

For the subset of pneumonia-related hospitalizations, the overall impact size of upcoding observed in our findings seems to be in line with a systematic literature search conducted by Lüngen and Lauterbach (2000), who estimated that upcoding was related with up to $1 \%$ of the inpatient care payments in Germany. However, this value is quite lower than the rates identified in a 1995-1996 coding audit in Australia, which revealed that an estimated $5.2 \%$ of the medical records were upcoded (Pongpirul and Robinson 2013). In the USA, it was found that one-third and onehalf of the case-mix increase occurred due to upcoding in the periods 1986-1987 and 1987-1988, respectively (Carter et al. 1990; Carter et al. 1991). In Portugal, Barros and Braun (2017) analyzed the same Portuguese database used in this study and found that upcoding has occurred in public hospitals to increase their budgets, but the financial impact was quantitatively small.

Concerning pneumonia-related conditions, a total of six discrepant codes presented similar effects on APR-DRG grouping as they drove the classification into the APRDRG 139 (see Table 2) when coded as principal diagnosis. Analyses based on SVM results showed that five out of the targeted six pneumonia-related conditions would have shifted these episodes to APR-DRG 137 when coded as secondary diagnosis (see Table 2), which is an APR-DRG with a higher complexity and reimbursement rate than APR-DRG 139. Thus, simply switching these conditions from principal to secondary diagnosis alone would result in more financial compensation to hospitals in the context of our case study. These cases should be watched more closely as they could be an indicator of upcoding practices, especially when a hospital presents a higher-than-expected frequency of such conditions coded as secondary diagnosis, rather than principal, relatively to its peers.

Comorbidities are secondary diagnoses with a high influence on SOI determination within the APR-DRG algorithm. In fifteen out of the seventeen evaluated comorbidities, at least one hospital presented a higher-than-expected frequency of cases. Adding more diagnoses, such as the patient's underlying comorbidities, is a typical upcoding practice, but only audit processes would determine whether the observed high frequencies are related to upcoding or whether 
other hospitals are underreporting comorbidities. It is important to highlight that the number of Charlson comorbidities by inpatient episode increased by $48 \%$ in the period of 2000-2010 in Portuguese hospital databases, though this increasing trend was not equal for all comorbidities, as the proportion of episodes with AIDS/HIV and peptic ulcer disease has decreased over the studied period (Freitas et al. 2016). However, the proportion of episodes with Charlson's myocardial infarction, dementia, and renal disease has increased above 100\% (Freitas et al. 2016). Those discrepancies in reporting certain comorbidities described by Freitas et al. (2016), which were also reported elsewhere in the literature among pneumonia hospitalizations (Chong et al. 2011), should be more closely monitored. In our case study, hospitals mostly differed in coding comorbidity renal disease, and a potential upcoding scenario should be investigated for hospital F, where this comorbidity alone drove the APR-DRG classification into a higher-paying APR-DRG in more than $40 \%$ of the episodes.

Overall, the number of cases flagged as upcoding by our method was proportionally small, and data credibility concerning upcoding was very high (see Table 4), indicating that data are credible enough and can be indiscriminately used for APRDRG grouping in the context of pneumonia-related hospitalizations. However, this may not be true for some diseases, namely, renal disease, which appears to have a considerable influence in APR-DRG grouping. In fact, this condition accounted for the highest percentage of potential upcoding cases, having thus an important impact on data credibility. In fact, according to our method, data of patients with renal disease would be considered not credible for hospitals B and F.

As a limitation of our study, we mention that flagging possible upcoding cases was based upon results obtained with the direct application of SVM models. Therefore, existing errors or shortcomings associated with the SVM models might have influenced or been replicated in our results. Furthermore, we only evaluated credibility related to coding the six conditions and fifteen comorbidities in which at least one hospital presented a significantly higher-thanexpected frequency of cases and did not consider possible coding issues related to other diagnoses or even inpatient procedures.

\section{Conclusion}

We identified potential upcoding in 2916 pneumonia inpatient episodes. We pose that measuring the level of credibility of the APR-DRG data allows hospital managers, providers, and stakeholders to better address this issue. In this article, we described and applied a method for credibility measurement regarding possible upcoding cases in pneumonia-related hospitalizations. According to our method, overall credibility levels were very high, and data can be considered credible and usable, except for data on renal disease. Hospitals significantly differed on reporting six pneumonia conditions and fifteen comorbidities, and we found a role of increasing APR-DRG complexity for these targeted codes, which thus should be investigated by means of audit. Furthermore, in the absence of historical audit data, our solution provided a fairly automated, generic, and reproducible method to measure data credibility for APR-DRG grouping, based upon well-established statistical and machine learning methods, which in turn could be applied to measure credibility concerning other types of coding errors, as well as for other diagnosis areas. 
Acknowledgments The authors would like to thank the Central Authority for Health Services, I.P. (ACSS) for providing access to the data. We would also like to thank to project GEMA: Generation and Evaluation of Models for Data Quality (Ref.: SBPLY/17/180501/000293) and the Master Program in Medical Informatics of the Faculty of Medicine and Faculty of Sciences of the University of Porto for financial support. Finally, we thank the project ECLIPSE (RTI2018-094283-B-C31), co-funded by the Spanish Ministry of Science, Innovation and Universities and Fundo Europeu de Desenvolvimento Regional (FEDER) funds.

\section{References}

Administração Central do Sistema de Saúde (2019). Benchmarking hospitais - grupos e instituições. http://benchmarking.acss.min-saude.pt/BH_Enquadramento/GrupoInstituicoes, .

Administração Central do Sistema de Saúde (2014). Agrupador de GDH All Patient Refined DRG. http://www2. acss.min-saude.pt/Portals/0/CN22.pdf. .

Aelvoet, W., Terryn, N., Windey, F., Redivo, M., van Sprundel, M., \& Faes, C. (2009). Miscoding: A threat to the hospital care system. How to detect it? Revue d'epidemiologie et de sante publique, 57(3), 169-177.

Aiello, F. A., \& Roddy, S. P. (2017). Inpatient coding and the diagnosis-related groups. Journal of Vascular Surgery, 66(5), 1621-1623.

Alonso, V., Santos, J. V., Pinto, M., Ferreira, J., Lema, I., Lopes, F., \& Freitas, A. (2019). Health records as the basis of clinical coding: Is the quality adequate? A qualitative study of medical coders' perceptions. Health Information Management Journal. https://doi.org/10.1177/1833358319826351.

Averill, R. F., McCullough, E. C., Goldfield, N. I., Hughes, J. S., Bonazelli, J., Bentley, L. (2013). 3M APRDRG classification system methodology overview, version 31. 3M health information systems. https://www. hcup-us.ahrq.gov/db/nation/nis/grp031_aprdrg_meth_ovrview.pdf. .

Barros, P. P., \& Braun, G. (2017). Upcoding in a national health service: The evidence from Portugal. Health Economics, 26(5), 600-618.

Carter, G. M., Newhouse, J. P., \& Relles, D. A. (1990). How much change in the case mix in-dex is DRG creep? Journal of Health Economics, 9(4), 411-428.

Carter, G. M., Newhouse, J. P., \& Relles, D. A. (1991). Has DRG creep crept up? Decomposing the case mix index change between 1987 and 1988. Santa Monica, California: RAND Corporation.

Centers for Medicare and Medicaid Services. (2014). International classification of diseases. Clinical Modification: Ninth Revision https://www.cms.gov/Medicare/Coding/ICD9ProviderDiagnosticCodes/codes.html. .

Centers for Medicare and Medicaid Services. (2019). International classification of diseases. Clinical Modification: Tenth Revision https:/www.cms.gov/Medicare/Coding/ICD10/index.html.

Chong, W. F., Ding, Y. Y., \& Heng, B. H. (2011). A comparison of comorbidities obtained from hospital administrative data and medical charts in older patients with pneumonia. BMC Health Services Research, 11, 105.

Chu, A., Ahn, H., Halwan, B., Kalmin, B., Artifon, E. L., Barkun, A., Lagoudakis, M. G., \& Kumar, A. (2008). A decision support system to facilitate management of patients with acute gastrointestinal bleeding. Artificial Intelligence in Medicine, 42(3), 247-259.

Dafny, L. S. (2005). How do hospitals respond to price changes? American Economic Review, 95(5), 1525-1547.

Di Giacomo, M., Piacenza, M., Siciliani, L., \& Turati, G. (2017). Do public hospitals respond to changes in DRG price regulation? The case of birth deliveries in the Italian NHS. Health Economics, 26, 23-37.

Feder, S. L. (2018). Data quality in electronic health records research: Quality domains and assessment methods. Western Journal of Nursing Research, 40(5), 753-766.

Freitas A., Lema I., da Costa-Pereira A. (2016) Comorbidity coding trends in hospital administrative databases. In: Rocha Á., Correia a., Adeli H., Reis L., Mendonça Teixeira M. (eds), New Advances in Information Systems and Technologies. Advances in intelligent systems and computing, vol 445. Springer, Cham.

Goodpasture, H., Nguyen-Dang, C., Lee, T. H., Ghazarian, P. G., \& Fulton, M. A. (2004). Miscoding as a cause of elevated simple pneumonia mortality. The Joint Commission Journal on Quality and Safety, 30(6), 335-341.

Hebert, P. L., McBean, A. M., \& Kane, R. L. (2005). Explaining trends in hospitalizations for pneumonia and influenza in the elderly. Medical Care Research and Review, 62(5), 560-582.

Hsia, D. C. (1990). Accuracy of Medicare reimbursement for cardiac arrest. Journal of the American Medical Association, 264(1), 59-62.

Hsia, D. C., Ahern, C. A., Ritchie, B. P., Moscoe, L. M., \& Krushat, W. M. (1992). Medicare reimbursement accuracy under the prospective payment system, 1985 to 1988. Journal of the American Medical Association, 268(7), 896-899.

ISO/IEC 25012 (2006). ISO/IEC 25012: Software product quality - Data quality model. https://iso25000. com/index.php/en/iso-25000-standards/iso-25012. 
Januleviciute, J., Askildsen, J. E., Kaarboe, O., Siciliani, L., \& Sutton, M. (2016). How do hospitals respond to price changes? Evidence from Norway. Health Economics, 25(5), 620-636.

Jarman, B., Gault, S., Alves, B., Hider, A., Dolan, S., Cook, A., Hurwitz, B., \& Iezzoni, L. I. (1999). Explaining differences in English hospital death rates using routinely collected data. British Medical Journal, 318(7197), 1515-1520.

Lau, E. C., Mowat, F. S., Kelsh, M. A., Legg, J. C., Engel-Nitz, N. M., Watson, H. N., Collins, H. L., Nordyke, R. J., \& Whyte, J. L. (2011). Use of electronic medical records (EMR) for oncology outcomes research: Assessing the comparability of EMR information to patient registry and health claims data. Clinical Epidemiology, 3, 259-272.

Lungen, M., \& Lauterbach, K. W. (2000). Upcoding: A risk for the use of diagnosis-related groups. Deutsche Medizinische Wochenschrift, 125(28-29), 852-856.

Luo, W., \& Gallagher, M. (2010). Unsupervised DRG upcoding detection in healthcare databases. In 2010 IEEE International Conference on Data Mining Workshops, Sydney, NSW (pp. 600-605).

Mathauer, I., \& Wittenbecher, F. (2013). Hospital payment systems based on diagnosis-related groups: Experiences in low- and middle-income countries. Bulletin of the World Health Organization, 91(10), 746-756.

Ministério da Saúde. (2017). Portaria n.o 207/2017 - Diário da República n.o 132/2017, série i de 2017-07-11. http://www.acss.min-saude.pt/wp-content/uploads/2016/12/Portaria_207_2017-1.pdf. .

Pimenta D., Souza J., Caballero I., Freitas A. (2019) Toward the measure of credibility of hospital administrative datasets in the context of DRG classification. In: Piattini M., Rupino da Cunha P., García Rodríguez de Guzmán I., Pérez-Castillo R. (eds) Quality of Information and Communications Technology. QUATIC 2019. Communications in Computer and Information Science, vol 1010. Springer, Cham.

Platt, J. (1998). Fast training of support vector machines using sequential minimal optimization. https://pdfs.semanticscholar.org/d1fa/8485ad749d51e7470d801bc1931706597601.pdf. Accessed 22 October 2019.

Pongpirul, K., \& Robinson, C. (2013). Hospital manipulations in the DRG system: A systematic scoping review. Asian Biomedicine, 7, 301-310.

Psaty, B. M., Boineau, R., Kuller, L. H., \& Luepker, R. V. (1999). The potential costs of upcoding for heart failure in the United States. The American Journal of Cardiology, 84(108-9), A9.

Quan, H., Sundararajan, V., Halfon, P., Fong, A., Burnand, B., Luthi, J.-C., Saunders, L. D., Beck, C. A., Feasby, T. E., \& Ghali, W. A. (2005). Coding algorithms for defining comorbidities in icd-9-cm and icd-10 administrative data. Medical Care, 43(11), 1130-1139.

Rea, S., Bailey, K. R., Pathak, J., Haug, P. J. (2013). Bias in recording of body mass index data in the electronic health record. AMIA Joint Summits on Translational Science Proceedings. AMIA Summit on Translational Science, 2013:214-218.

Reid, B., Allen, C., \& McIntosh, J. (2005). Investigation of leukaemia and lymphoma ar-drgs at a Sydney teaching hospital. Health Information Management, 34(2), 34-39.

Reid, B., Palmer, G., \& Aisbett, C. (2000). Under-coding in Australia limits the performance of drg groupers. Health Information Management, 29(3), 113-117.

Scott, I., Youlden, D., \& Coory, M. (2004). Are diagnosis specific outcome indicators based on administrative data useful in assessing quality of hospital care? BMJ Quality \& Safety, 13(1), 32-39.

Silverman, E., \& Skinner, J. (2004). Medicare upcoding and hospital ownership. Journal of Health Economics, 23(2), 369-389.

Singh, A., Thakur, N., \& Sharma, A. (2016). A review of supervised machine learning algorithms. In $20163 \mathrm{rd}$ international conference on computing for sustainable global development (INDIACom), New Delhi (pp. 1310-1315).

Sjoding, M. W., Iwashyna, T. J., Dimick, J. B., \& Cooke, C. R. (2015). Gaming hospital-level pneumonia 30-day mortality and readmission measures by legitimate changes to diagnostic coding. Critical Care Medicine, 43(5), 989-995.

Souza, J., Santos, J. V., Lopes, F., Viana, J., \& Freitas, A. (2018). Miscoding alerts within hospital datasets: An unsupervised machine learning approach. In A. Rocha, H. Adeli, L. P. Reis, \& S. Costanzo (Eds.), Trends and advances in information systems and technologies, Advances in intelligent systems and computing, vol (Vol. 746, pp. 1198-1207). Cham p: Springer.

Spangler, W. E., May, J. H., Strum, D. P., \& Vargas, L. G. (2002). A data mining approach to characterizing medical code usage patterns. Journal of Medical Systems, 26(3), 255-275.

Strong, D. M., Lee, Y. W., Wang, R. Y., Strong, D., Lee, Y. W., \& Wang, R. (1997). 10 potholes in the road to information quality. IEEE Computer, 30, 38-46.

Vapnik, V. (1995). The nature of statistical learning theory. New York, NY: Springer-Verlag.

Verplancke, T., Van Looy, S., Benoit, D., Vansteelandt, S., Depuydt, P., De Turck, F., \& Decruyenaere, J. (2008). Support vector machine versus logistic regression modeling for prediction of hospital mortality in 
critically ill patients with haematological malignancies. BMC Medical Informatics and Decision Making, 8 , 56.

Weiskopf, N. G., \& Weng, C. (2013). Methods and dimensions of electronic health record data quality assessment: Enabling reuse for clinical research. Journal of the American Medical Informatics Association, 20(1), 144-151.

Yates, D., Moore, D., \& McCabe, G. (1999). The practice of statistics. New York: Freeman.

Publisher's note Springer Nature remains neutral with regard to jurisdictional claims in published maps and institutional affiliations.

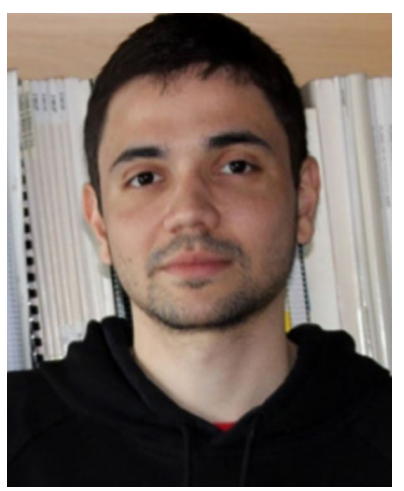

Julio Souza is a researcher at the CINTESIS (http://cintesis.eu/en/home/) as a member of the 2D4H research group (Secondary Data for Healthcare Research) and is currently working under a post- $\mathrm{PhD}$ (postdoc) scholarship in the project entitled "1st.IndiQare - Quality indicators in primary health care: validation and application of quality indicators as an evaluation and comparison tool" (http://cintesis.eu/en/portfolio-items/1st_ indiqare/). In 2019, he achieved a PhD degree in Clinical and Health Care Services Research at the Faculty of Medicine of the University in Porto, Portugal, with a thesis on the use of machine learning techniques to assess the quality of hospital administrative data used within Diagnosis-Related Groups (DRG) classification systems. He has been involved in research studies comprising several distinct areas related to health data science, namely health care quality indicators assessment, clinical coding, data quality and machine learning. He also collaborates with other partners from CINTESIS in studies related to topics such as biostatistics, health information management and public health.

\section{Affiliations}

\section{Julio Souza $^{1,2} \cdot$ Diana Pimenta $^{1} \cdot$ Ismael Caballero $^{3}$ - Alberto Freitas $^{1,2}$}

1 MEDCIDS - Department of Community Medicine, Information and Health Decision Sciences, Faculty of Medicine, University of Porto, Alameda Prof. Hernâni Monteiro, 4200-319 Porto, Portugal

2 CINTESIS - Center for Health Technology and Services Research, R. Dr. Plácido da Costa, 4200450 Porto, Portugal

3 Information Systems and Technologies Institute (ITSI), University of Castilla-La Mancha c\Moledores s/n, 13071 Ciudad Real, Spain 